\title{
THE TIP OF THE RED GIANT BRANCH AS A POPULATION II DISTANCE INDICATOR
}

\author{
SHOKO SAKAI \\ Jet Propulsion Laboratory \\ IPAC Caltech 100-22 Pasadena CA 91125 USA
}

\section{Introduction}

The period-luminosity (PL) relation of the Cepheid variable stars is the most reliable primary distance indicator for nearby galaxies, and serves as the foundation of the extragalactic distance scale, providing the firm calibration for various secondary distance indicators. However, a major disadvantage of the Cepheid distance scale is that it can only be applied to late-type systems with recent or current star formation. An alternative distance indicator is a Pop II, tip of the red giant branch (TRGB) method, which is extremely attractive because of its applicability to all morphological types of galaxies. As long as a metal-poor RGB stars are present in abundant numbers, the distance can be estimated with an accuracy that is as good as that of the Cepheid PL relation.

Baade (1944) first realized that the brightest resolved red stars in M31 and its two companion elliptical galaxies all had the same apparent brightness and color. Sandage (1971) later proposed that the brightest of the background "sheet" of red stars are those at the TRGB.

More recent studies have shown both observationally and theoretically that the TRGB method is potentially an excellent distance indicator (Frogel, Cohen \& Persson 1983, Da Costa \& Armandroff 1990 (DA90), Lee, Freedman \& Madore 1993 (LFM93)). The tip of the first-ascent red giant branch marks the helium ignition inside the degenerate core of low-mass stars. These stars evolve up the red giant branch, abruptly terminating their luminosity evolution at the TRGB, which can be detected as a discontinuity in the luminosity function, or in the color-magnitude diagram. In the $I$-band, the TRGB stars appear at $M_{I} \sim-4.0 \pm 0.1$, and this magnitude is insensitive to both metallicity and age for low-metallicity systems, a fea- 


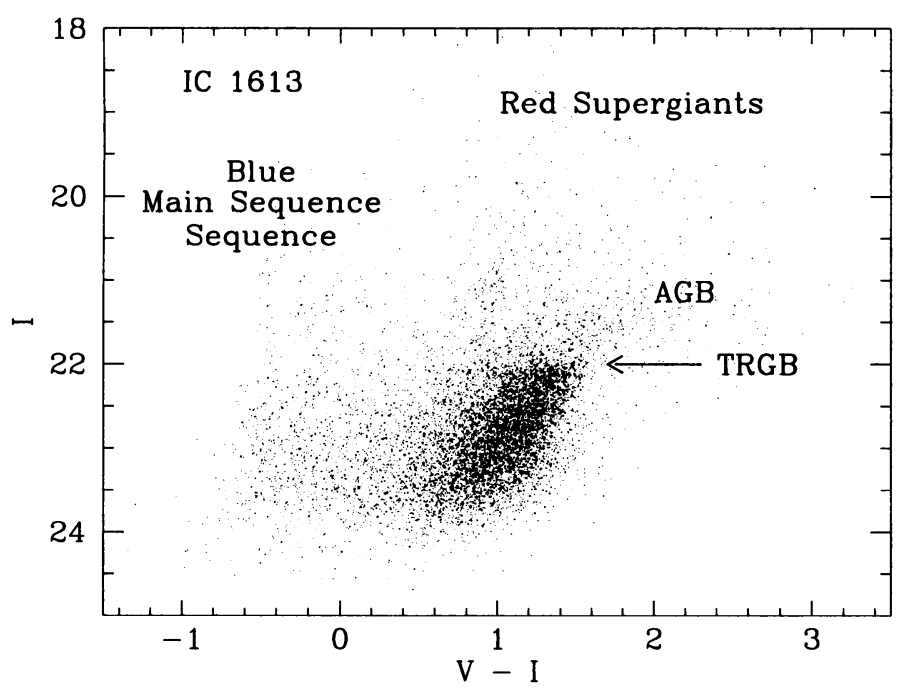

Figure 1. $I-(V-I)$ color-magnitude diagram of a Local Group dwarf irregular galaxy, IC 1613. The red and blue supergiants, as well as the AGB populations, are clearly observed. However, the most dominant feature is the red giant branch, with its distinct plateau representing the TRGB.

ture that makes the TRGB an useful distance indicator. The bolometric luminosity at the core helium flash for low-mass stars of the same metallicity varies only by $\sim 0.1 \mathrm{mag}$ for an age range of $2-15 \mathrm{Gyr}$ (Iben \& Renzini $1983)$, and also for metallicity range span by the Galactic globular clusters $(-2.2 \leq[\mathrm{Fe} / \mathrm{H}] \leq-0.7)$.

\section{TRGB Calibration}

The present calibration of the TRGB magnitude was first presented by DA90 and slightly modified by LFM93. It is fundamentally a calibration of the bolometric magnitude of the TRGB stars as a function of their metallicity. DA90 derived the calibration from $V I$ observations of RGB stars of six Galactic globular clusters, with metallicity range between $-2.2 \leq$ $[\mathrm{Fe} / \mathrm{H}] \leq-0.7$. The distances to the globular clusters were estimated using the metallicity $-M_{V}$ relation for RR Lyrae variable stars given by Lee, Demarque \& Zinn (1990: LDZ90) for $Y_{\mathrm{MS}}=0.23$.

The TRGB distance modulus is determined via the relation $(m-M)_{I}=$ $I_{\mathrm{TRGB}}-M_{\mathrm{bol}}+B C_{I}$. The bolometric correction was estimated by DA90 by comparing their optical photometry data with the extensive IR photometry of Frogel, Persson, \& Cohen (1983: FPC83) who had derived empirically the bolometric magnitudes for many globular cluster red giants by integrating the flux from $U B V J H K$ observations. Both the bolometric magnitude and 
correction terms are dependent on the metallicity and expressed as: $M_{\mathrm{bol}}=$ $-0.19[\mathrm{Fe} / \mathrm{H}]-3.81$ and $B C_{I}=0.881-0.243(V-I)_{\mathrm{TRGB}}$. The metallicity in turn is expressed in terms of the color of the red giant branch stars as: $[\mathrm{Fe} / \mathrm{H}]=-12.65+12.6(V-I)_{-3.5}-3.3(V-I)_{-3.5}^{2}$, where $(V-I)_{-3.5}$ is measured at the absolute $I$ magnitude of -3.5 .

The calibration introduced by DA90 is semi-empirical; it has adopted the LDZ90 distance scale which is based on the theoretical models of horizontal branch stars. Recently, Cassisi \& Salaris (1997) presented a theoretical calibration of the TRGB magnitude that utilized the canonical evolutionary models of stars for a combination of various masses and metallicities for $Y=0.23$ (see Salaris \& Cassisi 1996 for details). Cassisi \& Salaris (1997) find that the zero point of the theoretical calibration is $\sim 0.15 \mathrm{mag}$ brighter than the empirical zero point, while the slopes of the bolometric magnitude-metallicity relation remain in good agreement. They attribute this systematic difference to the small sample of stars observed by FPC83: statistically the probability that the brightest stars observed are actually TRGB stars is very small, implying that the empirical TRGB calibration would thus be underestimated.

Another source of systematic uncertainty in the TRGB distance scale is the uncertainties in the zero point of the RR Lyrae distance scale itself. The DA90/LFM93 calibration applied that of the LDZ90. However, there exist other RR Lyrae calibrations, such as the one derived by Carney et al. (1992) whose zero point is brighter by as much as $\sim 0.2 \mathrm{mag}$. Clearly, the issues pertaining to the calibration need to be explored in details in the near future.

\section{Recent Applications}

\subsection{QUANTITATIVE DETECTION OF THE TRGB}

Until recently, the position of the TRGB was estimated by visual inspection of the color-magnitude diagram. A quantitative method was introduced by LFM93 who applied a Sobel, edge-detection filter, which basically measures the first derivative, to a luminosity function histogram to estimate the TRGB position. The position of the tip is indicated by the highest peak in the filter output function. Sakai, Madore \& Freedman (1996) later modified the Sobel filter, such that it can be applied to a continuous, smoothed luminosity function.

\subsection{GROUND-BASED APPLICATIONS}

Madore \& Freedman (1995) studied the feasibility of the TRGB method as a precise distance indicator using the computer simulations, exploring 

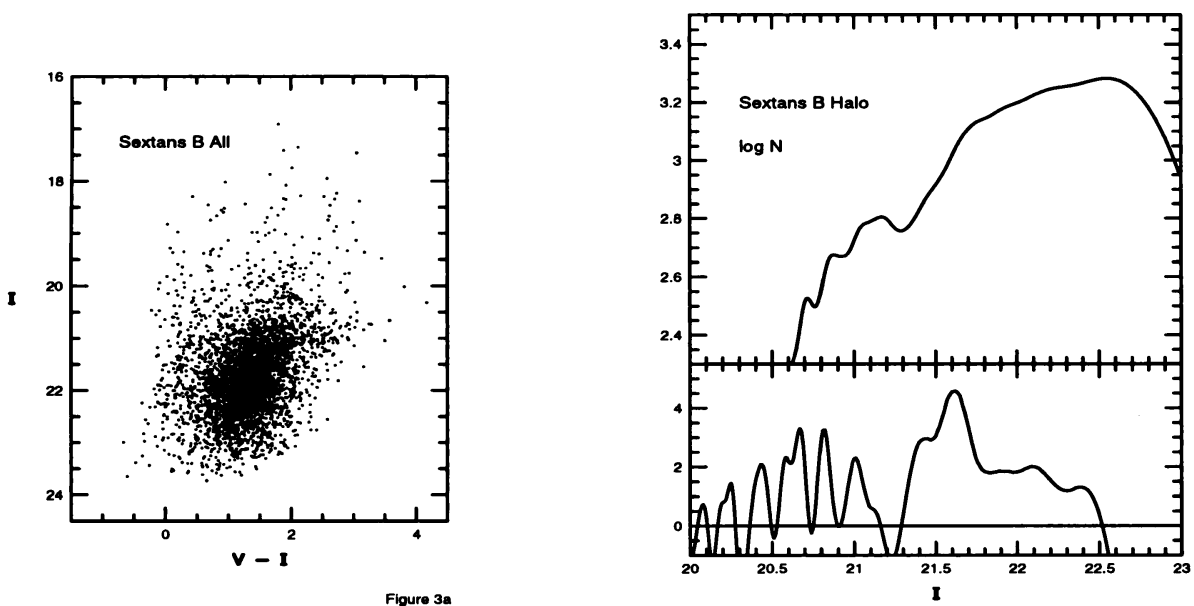

Figure 2. Color-magnitude diagram (right) and the logarithmic $I$-band luminosity function and Sobel filter output for a Local Group dwarf irregular galaxies, Sextans B.

several observational effects such as the signal to noise and crowding. They concluded that the TRGB method can be applied successfully out to at least $3 \mathrm{Mpc}$ using ground-based telescopes assuming $\leq 1$ arcsec seeing. Using the Hubble Space Telescope (HST), we expect that the distances out to $\sim 13 \mathrm{Mpc}$ can be measured for a typical exposure time of $\sim 8$ orbits.

Encouraged by this study, we have been undertaking a complete survey of TRGB distances to all galaxies in the Local Group and as far beyond it as the observational conditions allow. Using the Palomar $5 \mathrm{~m}$ and Las Campanas 2.5m telescopes, the data for most of the Local Group galaxies have been obtained so far, covering a wide range of morphological types. They include: M31 and its companions M33, M32, Andromeda dwarfs, WLM, IC 1613, IC 10. One example of the TRGB application to a Local Group galaxy is shown in Figure 2, in which a color-magnitude diagram and luminosity function for a dwarf irregular galaxy, Sextans B, are plotted. Below the luminosity function, an edge-detection filter output is plotted. We do successfully detect the tip at $I=21.60$, corresponding to a distance of $\mu=25.56 \pm 0.10$ after applying an extinction correction of $A_{I}=0.02$. This agrees well with the distance derived from the Cepheid variables (Sakai, Madore \& Freedman 1997). This example also shows that the TRGB method works even in dwarf irregular galaxies with dominant young stellar populations. 


\subsection{HST APPLICATIONS: BEYOND THE LOCAL GROUP}

The $H S T$ has also been used extensively to resolve red giant branch stars in nearby galaxies, as far out as $\sim 20 \mathrm{Mpc}$. Sakai et al. (1997) was successful in detecting the red giant branch population in the halo of a giant elliptical galaxy in Leo I Group at $11.5 \pm 1.6 \mathrm{Mpc}$, using the $I$-band luminosity function derived from the $H S T$ WFPC2 observations. Other reports of the TRGB detection using the $H S T$ include Centaurus A (NGC 5128) at 3.6 Mpc by Soria et al. (1996), and NGC 3115 at $11.0 \mathrm{Mpc}$ by Elson (1997). In M81 Group of galaxies, Caldwell et al. (1997) detected the red giants in two dwarf elliptical galaxies. Sakai, Madore \& Freedman (1997b) also observed the red giants in M82, an irregular galaxy in this group. Furthermore, some fields in Virgo cluster were HST targets for the detection of the RGB population: Ferguson et al. (1997) reported the detection of intergalactic RGB in the Virgo cluster at 18.2 Mpc, while Harris et al. (1997) reports the detection of the TRGB in a dE galaxy, VCC 1104, also in Virgo.

\section{Metallicity Dependence of the Cepheid PL Relation}

The Cepheid distance scale has served as the cornerstone in the extragalactic distance scale. Recently, the extragalactic distance scale as a whole has begun seeing a remarkable convergence. This in turn has made it increasingly important to understand the systematic uncertainties in the Cepheid distance scale itself. One of the outstanding sources of systematic errors is the metallicity dependence of the Cepheid variable stars. Several studies have argued for a significant metallicity dependence (e.g. Gould 1994, Beaulieu et al. 1997, Kochanek 1997, Sasselov et al. 1997). We have compiled a database of galaxies whose distances have been measured by both the Cepheid PL relation and the TRGB method (Kennicutt et al. 1998). Because the TRGB magnitude is remarkably insensitive to the metallicity, the difference between the TRGB and Cepheid distances can be plotted as a function of the Cepheid metallicity to test for the existence of any trend. Figure 3 shows the correlation. The metallicity shown here were derived from the observations of HII regions. Three lines indicate the metallicity dependence $\left(\gamma=\delta(m-M)_{0} / \delta[O / H]\right)$ derived by Kennicutt et al. 1998 (0.24 mag dex ${ }^{-1}$ ) from the $H S T$ observations of Cepheids in two fields in M101, Beaulieu et al. 1997 and Kochanek 1997 (0.44 mag dex $\left.{ }^{-1}\right)$ and Gould 1994 ( $\left.0.88 \mathrm{mag} \mathrm{dex}^{-1}\right)$. The uncertainties in the zero point calibration of the TRGB distance scale due to, for example, the RR Lyrae distance scale uncertainties, would affect the zero point of this relation in Figure 3, but the slope should remain unchanged. The TRGB-Cepheid comparison test offer strong evidence for the weak metallicity dependence of the Cepheid PL relations. 


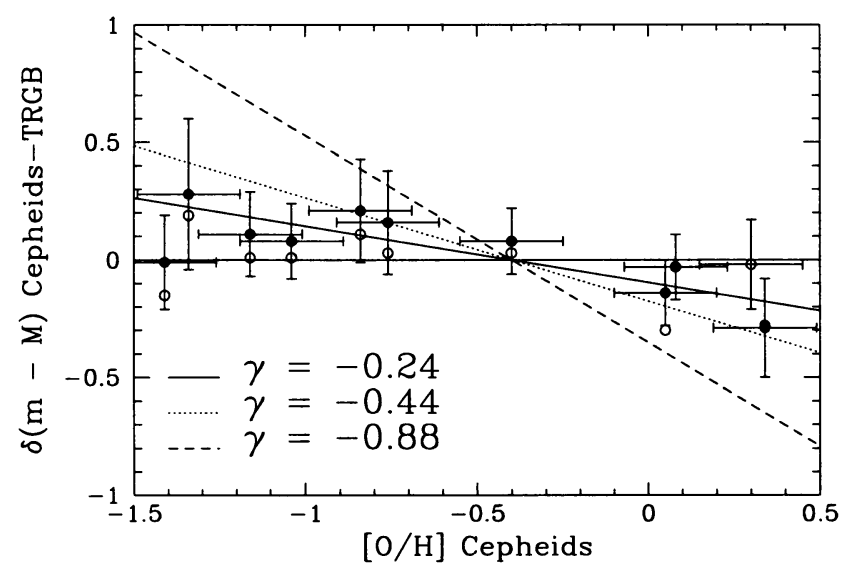

Figure 3. The differences between the TRGB and Cepheid distances for nearby galaxies are shown as a function of Population I metallicity derived from the HII region observations. This offers strong evidence for the weak metallicity dependence of the Cepheid PL relations. Adopted from Kennicutt et al. (1998).

I would like to thank Barry Madore and Wendy Freedman for their invaluable contributions to much of the work presented here. I would like to also thank the support from the NASA LTSA Program, NAS7-1260.

\section{References}

Baade, W., 1944, ApJ, 100, 137

Beaulieu, J.P. 1997, A\&A, 318, L47

Caldwell, N., Armandroff, T.E., Da Costa, G.S. \& Seitzer, P., 1997, AJ, in press

Carney, B.W., Storm, J., \& Jones, R.V., 1992, ApJ, 386, 663

Da Costa, G. S. \& Armandroff, T. E., 1990, AJ, 100, 162

Elson, R.A.W., 1997, MNRAS, 286, 771

Ferguson, H., von Hippel, T. \& Tanvir, N.I., 1997, in press

Frogel, J.A., Cohen, J.G. \& Persson, S.E., 1983, ApJ, 275, 773

Gould, A., 1994, ApJ, 426, 542

Harris, W.E., Durrell, P.R., Pierce, M.J. \& Secker, J., 1997, in preparation

Iben, I. \& Renzini, A. 1983, ARAA, 21, 271

Kennicutt et al. 1998, ApJ, in press

Kochanek, C.S., 1997, ApJ, in press

Lee, M. G., Freedman, W. L. \& Madore, B. F., 1993, ApJ, 417, 553

Lee, Y.-W., Demarque, P. \& Zinn R., 1990, ApJ, 350, 155

Madore, B. F. \& Freedman, W. L., 1995, AJ, 109, 1645

Sakai, S., Madore, B. F., \& Freedman, W. L., 1996, ApJ, 461, 713

Sakai, S., Madore, B. F., Freedman, W. L., Lauer, T. R., Ajhar, E. A. \& Baum, W. A., 1997, ApJ, 478, 49

Sakai, S., Madore, B.F., \& Freedman, W.L., 1997, ApJ, 480, 589

Salaris, M. \& Cassisi, S., 1996, AA, 305, 858

Salaris, M. \& Cassisi, S., 1997, MNRAS, 289, 406

Sandage, A.R., 1971, in Nuclei of Galaxies, ed. D.J.K.O'Connell

Soria R. et al., 1996, ApJ, 465, 79 